\title{
Additional Information Regarding the SEVITENSION Study
}

Luis Ruilope

To view enhanced content go to www.advancesintherapy.com

Received: July 21, 2014 / Published online: August 22, 2014

(C) The Author(s) 2014. This article is published with open access at Springerlink.com

Dear Dr. Menown,

I am pleased to report that we have received considerable interest since the publication of our article (Ruilope L, Schaefer A. The fixed-dose combination of olmesartan/amlodipine was superior in central aortic blood pressure reduction compared with perindopril/ amlodipine: a randomized, double-blind trial in patients with hypertension. Adv Ther. 2013;30:1086-99 [1]) last December. The results confirmed the effects of combined renin angiotensin system and calcium channel blockade on lowering central blood pressure. Furthermore, it extended these findings by showing that the angiotensin II receptor blocker olmesartan medoxomil could produce similar changes in central blood pressure when combined with a calcium channel blocker.

One enquiry concerned the levels of baseline blood pressure in the treatment groups

L. Ruilope ( $\square)$

Department of Preventive Medicine and Public Health, Universidad Autonoma, Madrid, Spain

e-mail: ruilope@ad-hocbox.com

L. Ruilope

Hypertension Unit, Hospital 12 de Octubre, 28041

Madrid, Spain described in the report. We are happy to provide these baseline values here, but feel that it is advisable to first remind readers of the context in which these data should be viewed. The use of a 4 -week run-in period before the start of double-blind treatment meant that all patients had been treated with amlodipine for at least 4 weeks (Table 1 footnotes). Some patients would have received amlodipine $5 \mathrm{mg}$ for the first 2 weeks of the run-in period and then been uptitrated to amlodipine $10 \mathrm{mg}$ for the final 2 weeks. Furthermore, some patients had also received other antihypertensive agents for up to 2 weeks before the start of doubleblind treatment and some had received amlodipine $10 \mathrm{mg}$ for more than 2 weeks. Amlodipine is an effective antihypertensive agent and even though patients entered double-blind treatment only if their blood pressure control was inadequate, the treatments used prior to double-blind treatment would have already produced significant reductions in blood pressure. This should be remembered when looking at the baseline data (Table 1). Also, as specified in the study protocol, the per protocol set was used to assess changes in the primary variable (central 
Table 1 Baseline hemodynamic characteristics of patients who entered double-blind treatment

\begin{tabular}{llll}
\hline Characteristics & $\begin{array}{l}\text { OLM/AML } \\
(\mathbf{4 0 / 1 0 ~} \mathbf{~ m})\end{array}$ & $\begin{array}{l}\text { PER/AML } \\
(\mathbf{8} / \mathbf{1 0} \mathbf{~ m g})\end{array}$ & Total \\
\hline Per protocol set & $n=198$ & $n=192$ & $n=390$ \\
Central SBP, mmHg & $135.3(11.57)$ & $135.2(10.89)$ & $135.3(11.22)$ \\
Full analysis set & $n=221$ & $n=221$ & $n=442$ \\
Seated SBP, mmHg & $148.5(10.30)$ & $148.9(11.93)$ & $148.7(11.13)$ \\
24-h ambulatory SBP, mmHg & $134.8(11.62)$ & $133.3(11.28)$ & $134.0(11.46)$ \\
Daytime ambulatory SBP, mmHg & $138.3(12.10)$ & $137.1(11.58)$ & $137.7(11.84)$ \\
Night-time ambulatory SBP, mmHg & $126.9(12.72)$ & $125.0(13.02)$ & $125.9(12.89)$ \\
Central DBP, mmHg & $86.8(9.30)$ & $87.3(9.68)$ & $87.1(9.48)$ \\
Seated DBP, mmHg & $85.8(8.92)$ & $86.1(9.43)$ & $86.0(9.17)$ \\
24-h ambulatory DBP, mmHg & $79.2(8.76)$ & $79.2(8.36)$ & $79.2(8.55)$ \\
Daytime ambulatory DBP, mmHg & $82.0(9.31)$ & $82.1(8.44)$ & $82.0(8.88)$ \\
Night-time ambulatory DBP, mmHg & $73.1(8.76)$ & $73.1(9.52)$ & $73.1(9.14)$ \\
\hline
\end{tabular}

Values are mean (standard deviation)

Before starting double-blind treatment patients entered a 4-week run in, unless they had been receiving amlodipine $10 \mathrm{mg}$ as monotherapy before the study (in which case they entered double-blind treatment directly). Patients who had been receiving drug treatment before the study continued with their former medication (except for calcium channel blockers) during the first 2 weeks of the run in and also received amlodipine $5 \mathrm{mg}$ once daily. During the final 2 weeks of the run in, all former medications were withdrawn and patients received amlodipine $10 \mathrm{mg}$ as their only treatment. Patients entered double-blind treatment if their seated systolic blood pressure/diastolic blood pressure was $\geq 140 / 90 \mathrm{mmHg}(\geq 130 / 80 \mathrm{mmHg}$ for diabetes or CKD). For a description of the per protocol and full analysis sets, please see Ruilope and Schaefer [1] $A M L$ amlodipine, $D B P$ diastolic blood pressure, $O L M$ olmesartan, $P E R$ perindopril, $S B P$ systolic blood pressure

systolic blood pressure) and the full analysis set was used for analysis of all other parameters.

Yours sincerely,

Professor Luis Ruilope

\section{ACKNOWLEDGMENTS}

This study was sponsored by Daiichi Sankyo Europe GmbH, Munich, Germany. Prof. Dr. Luis Ruilope was the co-ordinating investigator and acted as the medical expert for the study. Medical writing assistance during the preparation of this manuscript was funded by
Daiichi Sankyo Europe GmbH and provided by Phil Jones, PhD, of inScience Communications, Springer Healthcare, Chester, UK. Professor Luis Ruilope is the guarantor for this article, and takes responsibility for the integrity of the work as a whole. The author wishes to express his gratitude to the investigators, study nurses and co-ordinators who were involved in the SEVITENSION study. The SEVITENSION Study Investigators were: Jose Abellan, Pedro Aranda, Cesar Cerezo, Jose Antonio Divison, Luis Garcia Ortiz, Juan Garcia Puig, Pablo Gomez, Jorge Gomez Cerezo, Nieves Martell, Anna Oliveras, Enrique Rodilla, Jose Saban, Julian Segura, Carmen Suarez and Luis Vigil. 
Conflict of interest. Prof. Dr. Ruilope has served as an advisor and speaker for Daiichi Sankyo.

Compliance with ethics guidelines. The study and all procedures were conducted in accordance with the Helsinki Declaration of 1975, and the 2000 and 2008 revisions, and the International Conference on Harmonisation E6 Guidelines for Good Clinical Practice, the European Commission Directive 2001/20 EC, the European Commission Directive 2005/28 EC and the European Commission Directive $95 / 46$ EC. The study was approved by relevant Independent Ethics Committees or Institutional Review Boards. Informed consent was obtained from all patients for being included in the study.
Open Access. This article is distributed under the terms of the Creative Commons Attribution Noncommercial License which permits any noncommercial use, distribution, and reproduction in any medium, provided the original author(s) and the source are credited.

\section{REFERENCE}

1. Ruilope L, Schaefer A. The fixed-dose combination of olmesartan/amlodipine was superior in central aortic blood pressure reduction compared with perindopril/ amlodipine: a randomized, double-blind trial in patients with hypertension. Adv Ther. 2013;30: 1086-99. 